\title{
1 Human HspB1, HspB3, HspB5 and HspB8: Shaping these Disease Factors during \\ 2 Vertebrate Evolution
}

3 Rainer Benndorf, Ryan Velazquez ${ }^{1}$, Jordan D. Zehr' ${ }^{1}$, Sergei Kosakovsky Pond ${ }^{1}$, Jody L. Martin ${ }^{2}$,

4 Alexander G. Lucaci ${ }^{1}$

6 address of the corresponding author: Jody L. Martin, Cell and Molecular Core, Cardiovascular

7 Research Institute, University of California at Davis, Davis, California, USA, E-mail:

8 jodmartin@ucdavis.edu

Rainer Benndorf (Plauen, Germany) is retired.

12 Abstract

13 Small heat shock proteins (sHSPs) emerged early in evolution and occur in all domains of life and nearly in all species, including humans. Mutations in four sHSPs (HspB1, HspB3, HspB5, HspB8) are associated with neuromuscular disorders. The aim of this study is to investigate the evolutionary forces shaping these sHSPs during vertebrate evolution. We performed comparative evolutionary analyses on a set of orthologous sHSP sequences, based on the ratio of non-synonymous : synonymous substitution rates for each codon. We found that these sHSPs had been historically exposed to different degrees of purifying selection, decreasing in this order: HspB8 $>$ HspB1, HspB5 $>$ HspB3. Within each sHSP, regions with different degrees of purifying selection can be discerned, resulting in characteristic selective pressure profiles. The conserved $\alpha$-crystallin domains were exposed to the most stringent purifying selection compared to the flanking regions, supporting a 'dimorphic pattern' of evolution. Thus, during vertebrate evolution the different sequence partitions were exposed to different and measurable degrees of selective pressures. Among the disease-associated mutations, most are missense mutations primarily in HspB1 and to a minor extent in the other sHSPs. Our data provide an explanation for this disparate incidence. Contrary to the expectation, most missense mutations cause dominant disease phenotypes. Theoretical considerations support a connection between the historic exposure of these sHSP genes to a high degree of purifying selection and the unusual prevalence of genetic dominance of the associated disease phenotypes. Our study puts the genetics of inheritable sHSP-borne diseases into the context of vertebrate evolution.

11 Institute for Genomics and Evolutionary Medicine, Department of Biology, Temple University,

2 Philadelphia, PA 19122, USA

32 Cell and Molecular Core, Cardiovascular Research Institute, University of California at Davis, Davis, 


\section{Introduction}

32 The human genome encodes ten small heat shock proteins (sHSP) (Fontaine et al. 2003; Kappé et al.

33 2003), now systematically named HspB1 through HspB10, whether or not their expression is induced

34 by stress factors (Kampinga et al. 2009). The defining feature of sHSPs is a conserved $\sim 85$ amino acid

35 long sequence stretch called the a-crystallin domain (aCD) (de Jong et al. 1998; Kappé et al. 2010;

36 Mymrikov et al. 2011). It is believed that the primary function of sHSPs is to act as holdase

37 chaperones with a depot function for unfolded proteins, thereby shaping protein-protein interactions.

38 This, in turn, controls many cellular processes and structures such as cell signaling, cell architecture,

39 differentiation, redox homeostasis, apoptosis, and others (Carra et al. 2019).

sHSPs occur in all domains of life, including Archea, Bacteria, and Eucarya, suggesting that

41 they emerged early in evolution. Nearly all organisms contain genes encoding sHSPs except a few

42 pathogens that have lost these genes (Kriehuber et al. 2010). The number of paralogous sHSP genes

43 per genome can vary between 2 (average) in Archea and Bacteria, 3 (average) in fungi, 8 (average) in

44 Metazoa, and up to 50 (maximum) in some plants (Kriehuber et al. 2010). Many vertebrates, including

45 humans, contain ten paralogous sHSP genes, although some variation may occur, notably in the fish

46 taxa. Among the vertebrate sHSPs, orthology to the human sHSPs can be easily established by

47 phylogenetic analysis in most cases, whereas this is usually not possible across the other taxa.

Multiple sequence alignment (Fontaine et al. 2003; Franck et al. 2004; Kriehuber et al. 2010)

49 reveals that the conserved $\alpha \mathrm{CD}$ is flanked by regions of elevated sequence variability: the moderately

50 conserved N-terminal region (NTR) and the highly variable C-terminal extension (CTE). Sometimes a

51 short central region (CeR) with high sequence variability, positioned between the NTR and the $\alpha \mathrm{CD}$

52 (possibly serving as a 'hinge' between both regions), is considered separately (Wang et al. 2000;

53 Fontaine et al. 2003). This organization of the primary structure is summarized in Fig. S1. The aCD

54 contains several $\beta$-strands forming two anti-parallel $\beta$-sheets (sandwich structure), whereas the 
55 flanking regions (NTR, CeR, CTE) are less ordered or even disordered (Carra et al. 2019; Webster et al.

56 2019; Boelens 2020). Whether or not such regions without a compact folding structure should be

57 considered domains is debatable. Together, these domains and regions contribute to both the dynamic

58 association of the monomers into high molecular mass complexes and to their holdase chaperone

59 function.

The evolutionary history of sHSPs through all domains of life is characterized by certain unique

61 features (Kriehuber et al. 2010): (1) According to the PFAM classification ${ }^{3}$, the overwhelming majority of sHSPs from all domains of life contain just one domain, the highly conserved aCD, contrary to other studied protein superfamilies which typically diverged by recruiting additional domains; (2) The evolution of sHSPs follows a 'dimorphic pattern': the aCD has a monophyletic origin reflecting the evolution of species, as opposed to the flanking regions which were historically remodeled several times in parallel but independent of the $\alpha C D$. Thus, the $\alpha C D$ and the flanking regions have a fundamentally different evolutionary history. It is believed that this combination of the conserved aCD with the variable flanking regions (NTR, CeR, CTE) provides a unique and high degree of structural and functional speciation.

Four of these sHSP genes carry known mutations which have been associated with

71 neuromuscular disease phenotypes in humans: HspB1 (Hsp27, Hsp25), HspB3, HspB5 (aB-crystallin), and HspB8 (Hsp22) (Datskevich et al. 2012; Benndorf et al. 2014; Vendredy et al. 2020), not counting polymorphisms or other sequence variants with unclear relation to disease. Mutant alleles of HspB1,

$74 \mathrm{HspB} 3$ and HspB8 typically cause neuropathies with a spectrum of symptoms ranging from the clinical 75 characteristics 'Distal Hereditary Motor Neuropathy' and 'Charcot-Marie-Tooth Disease' to some forms 76 of 'Amyotrophic Lateral Sclerosis' and of myopathies (Table S1; Benndorf et al. 2014; Echaniz-Laguna 77 et al. 2017; Adriaenssens et al. 2017; Katz et al. 2020; Chen et al. 2021), tentatively suggesting a 78 functional overlap of these sHSPs. However, the pathogenicity of the HspB3 mutations has been

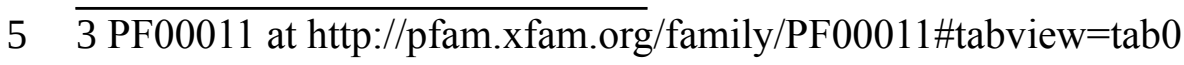


79 recently disputed (Adriaenssens et al. 2017; Vendredy et al. 2020). Quite differently, mutations in

80 HspB5 cause exclusively various forms of myopathies, including cardiomyopathies, and cataracts in

81 the lens of the eye, the latter partially in association with the myopathies. The overwhelming majority

82 among these disease-associated mutations are missense mutations, as opposed to a small number of

83 identified frame shift, nonsense and elongation mutations (Benndorf et al. 2014; Vendredy et al. 2020).

84 Disorders caused by the mutant sHSP alleles with their high penetrance belong to the group of

85 Mendelian diseases (Quintana-Murci and Barreiro 2010), in spite of the fact that in most patients the

86 symptoms develop only later in life (late onset diseases). Most of these missense mutations are

87 associated with a dominant disease phenotype, or this association can be assumed, e.g., when the

88 mutations occur sporadically (Table S1). Four missense mutations in HspB1 and HspB5 are associated

89 with a recessive disease phenotype.

One approach for understanding the evolutionary forces that have shaped proteins is to measure

91 the ratio $(\omega)$ of the non-synonymous $(\beta)$ and synonymous $(\alpha)$ substitution rates $(\omega=\beta / \alpha)$ at each

92 codon position in a gene of interest (Kosakovsky Pond and Frost 2005). Non-synonymous

93 substitutions change the amino acid being coded for at that site, which can directly affect protein

94 structure and influence the fitness of an organism through negative (purifying) selective pressure. In

95 contrast, synonymous substitutions do not change the amino acid being coded for, leaving the amino

96 acid sequence unchanged. Synonymous substitutions are typically viewed as neutral and provide a

97 baseline rate against which non-synonymous evolutionary rates can be calibrated. Therefore, the ratio

$98 \omega$ of relative rates of non-synonymous and synonymous substitutions can provide information as to the

99 type of selection that has acted upon a given set of protein-coding sequences, upon selected sequence

100 segments (e.g. structural domains), or upon single codons (e.g. mutation sites). This ratio $\omega$ has

101 become a standard measure of selective pressure in evolutionary biology (Frost et al. 2005; Arenas

102 2015). When there are more non-synonymous changes relative to synonymous changes, the $\omega$ ratio

103 will be greater than 1, indicative of positive, diversifying selection. Conversely, if there are fewer non- 
104 synonymous changes relative to synonymous changes, the $\omega$ ratio will be less than 1 , indicative of 105 negative, purifying selection.

106 In this study we have estimated the ratio $\omega$ for each codon along the entire length of the 107 sequences of human HspB1, HspB3, HspB5, and HspB8, using the aligned vertebrate sequences of the 108 orthologs. We provide evidence for the exposure of the NTR, $\alpha \mathrm{CD}, \mathrm{CeR}$, and CTE in these sequences 109 to different degrees of purifying selection during vertebrate evolution. This disparate pattern of 110 selective pressure within vertebrates fits well to the wider pattern of the dimorphic evolution that has 111 shaped sHSPs across all domains of life, i.e., from the origin of the tree of life onward (Kriehuber et al. 112 2010). Thus, the same forces that shaped the sHSPs early in evolution are detectable also in the 113 vertebrate evolution in recent 400-500 million years. Although similar, the $\omega$ profiles of the four 114 studied human sHSPs exhibit differences indicating exposure of each sHSP to variable degrees of 115 evolutionary pressure. For the disease-associated missense mutation sites, we find that all sites were 116 historically exposed to detectable purifying selection, although to different degrees. Additionally, our 117 data provide an explanation for the different incidence by which the affected sHSPs harbored disease118 associated mutations.

\section{Methods}

\section{Retrieval of vertebrate sHSP sequences from the databases}

121 This study was restricted to the vertebrate sHSP sequences, specifically to the taxon Gnathostomata. 122 Gnathostomata emerged more than 400 million years ago in evolution (Kuraku et al. 2016) and 123 comprises all vertebrate species except those from the taxon Agnatha. Gnathostomata orthologs of 124 human HspB1, HspB3, HspB5, and HspB8 were retrieved by a BLAST-P search at the NCBI website ${ }^{4}$, 125 using the full-length human protein sequences as the query sequence. Additionally, several sequences

\footnotetext{
64 https://www.ncbi.nlm.nih.gov/
} 
126

127

128

129

130

\section{Inclusion criteria}

132 Each retrieved candidate protein sequence was aligned to the human ortholog using the EMBOSS-

133 Needle algorithm ${ }^{7}$, returning the degree of identity and similarity of each sequence to be tested with the

134 human sequence. Sequences exhibiting $\geq 50 \%$ identity and $\geq 60 \%$ similarity were included in further

135 analysis, with all other sequences being excluded. When aligned by the EMBOSS-Needle algorithm, a

136 few candidate sequences exhibit a putative N-terminal extension, compared to the human orthologs and

137 to the orthologs of nearly all other species. However, these putative N-terminal extensions were

138 deduced in silico by algorithms based on DNA-sequencing data without any experimental support.

139 Therefore, these putative N-terminal extensions can be assumed to result from an erroneous in silico-

140 identification of the initiation codons and were consequently excluded from further processing. In

141 these sequences, we used the canonical initiation codons based on the alignment with the respective

142 human sHSPs.

143

144 trees using alignment and phylogenetic inference (Kristensen et al. 2011) at the Clustal Omega web

145 site $^{8}$. Phylogenetic trees were constructed using all ten human sHSPs and the query sequence.

146 Orthology was determined through analysis of patterns of sequence clustering within the reconstructed

147 phylogenetic tree. Sequences were labeled as orthologs of human HspB1, HspB3, HspB5 or HspB8 if

$7 \quad \overline{5 \text { https://useast.ensembl.org/index.html?redirect }=\text { no }}$

86 https://web.expasy.org/translate/

97 https://www.ebi.ac.uk/Tools/psa/emboss_needle/

108 https://www.ebi.ac.uk/Tools/msa/clustalo/ 
148 they grouped with these sHSPs, whereas sequences that did not group with these sHSPs were

149 discarded.

150 Applying the criteria above resulted in the exclusion of several candidate sHSP-like sequences,

151 notably from the fish taxa. The numbers of sHSP sequences that finally entered analyses for the 152 detection of natural selection within the taxon Gnathostomata were 152 for HspB1, 130 for HspB3, 153147 for HspB5, and 144 for HspB8 (Table S2).

\section{Species included in the sHSP sequence analysis}

155 The designation of species and their taxonomic ranking was according to the NCBI web site ${ }^{9}$. This 156 study includes sHSP sequences from Gnathostomata (Vertebrata: Gnathostomata) species representing 157 the major vertebrate taxa: Mammalia (Gnathostomata: Teleostomi: Euteleostomi: Sarcopterygii:

158 Dipnotetrapodomorpha: Tetrapoda: Amniota: Mammalia; includes primates, rodents, lagomorphs, 159 ungulates, whales, bats, shrews, carnivorians, mammals of African origin, marsupials, armadillos, 160 others), Sauria (Amniota: Sauropsida; Sauria; includes crocodiles, lizards, snakes, birds, turtles), 161 Amphibia (Tetrapoda: Amphibia; includes frogs and toads), Coelacanthimorpha (Sarcopterygii: 162 Coelacanthimorpha; includes just one species, the coelacanth), Neopterygii (Euteleostomi: 163 Actinopterygii: Neopterygii, includes various taxa of ray-finned fish), and Chondrichthyes 164 (Gnathostomata: Chondrichthyes or cartilaginous fish). The names of all species included in this study 165 and their taxonomic ranking are given in Table S2.

166 Determination of codon-specific evolutionary pressure along the entire length of the human 167 sHSPs

168 The strength and direction of pervasive natural selection (i.e., purifying versus positive or diversifying) 169 was assessed at the level of individual codon sites in each of the four sHSPs using the Fixed Effects

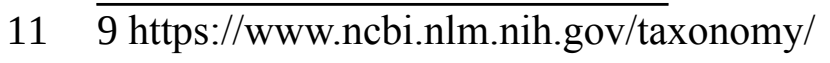


170 Likelihood (FEL) method implemented in the HyPhy package (Pond et al. 2005) and the Datamonkey ${ }^{10}$

171 web application (Pond and Frost 2005). A dN/dS point estimate with confidence intervals was

172 computed for each site using the FitMG94 model (Kosakovsky Pond et al. 2010). The confidence

173 interval around the maximum likelihood point estimate of $\omega$ was derived by computing occurrences

174 where the parameter estimate was not rejected in favor of the maximum likelihood estimate at a defined

175 significance level (95\% confidence of profile likelihood). A phylogenetic tree was constructed, using

176 FastTree2.1 (Price et al. 2010) with the Generalized Time Reversible (GTR) model of nucleotide

177 evolution. We estimated the ratio $(\omega)$ of non-synonymous $(\beta)$ to synonymous $(\alpha)$ substitution rates for

178 each codon along the entire multiple sequence alignment by maximum likelihood. These $\omega$-values are

179 a commonly used measure of selection, expected to be i) significantly (using the likelihood ratio test,

180 LRT) less than one when purifying selection is predominant, ii) not significantly different from one for

181 neutral evolution, and iii) significantly greater than one if positive selection is predominant. We report

$182 \omega$-value estimates for all codons of the human sHSP sequences and omit all codons in vertebrate

183 sequences that have no homologous sites in the human sequences (Table S3). The estimated numeric

$184 \omega$-values also indicate the strength of the selective pressure for a given codon, e.g., with values of 0 or

185 near 0 suggesting a greater degree of purifying selection. p-values indicating statistical significance

186 were derived using the asymptotic chi-square test distribution for the LRT. Statistical significance

187 (LRT p-value $\leq 0.1)$ for detecting purifying selection was observed for all codons with $\omega=0$ (no

188 columns; cf. Fig. 1) and typically for most codons with $0<\omega<0.5$ (indicated by black columns).

189 Statistical significance (LRT p-value $\leq 0.1$ ) for detecting diversifying selection was observed for two

190 codons in HspB3 with $\omega>1.5$ (indicated by blue columns). For codons with $\omega \approx 1$, no selective force

191 was detected (neutral evolution; indicated by gray columns). Table S3 gives the numeric values of $\beta$,

$192 \alpha, \omega(\mathrm{dN} / \mathrm{dS}$ point estimates), and the $\mathrm{dN} / \mathrm{dS}$ confidence intervals with the upper and lower bounds.

10 https://www.datamonkey.org 
194 initiator methionines is not defined $(\beta / \alpha ; \beta=0, \alpha=0)$. Depending on the alignments, the $\omega$-values for

195 several internal methionine and tryptophan (encoded solely by TGG) residues, or occasionally for other

196 amino acid residues within sequences may also be undefined $(\beta / \alpha ; \beta=0, \alpha=0)$ or approaching infinity

$197(\beta / 0 ; \beta / \alpha$ with $\alpha \rightarrow 0)$. These codons (marked in Table S3 and by columns of gray $x$ in Fig. 1) which

198 were included in the FEL analysis were excluded from the subsequent selection analyses using the

199 FitMG94 method (see below), while other internal, variable methionine and tryptophan residues were 200 included in both analyses.

\section{Sequence partitions}

202 For comparison of the selective pressures acting historically on different parts of the sHSP sequences,

203 the human sequences were separated into defined partitions. These partitions were primarily inferred

204 from the profiles of the $\omega$-values as returned by the FEL-method (cf. Fig. 1), thereby separating regions

205 with relatively high, medium, or low average $\omega$-values. The obtained partitions correspond

206 approximately, though not perfectly, to the domains and regions as identified earlier by multiple

207 alignment of the sHSPs (cf. Fig. S1): The NTR, $\alpha \mathrm{CD}, \mathrm{CTE}$, and CeR can be discerned in most cases.

208 Where this $\omega$-plot-based discrimination was not possible (e.g., for some regions of HspB5), multiple

209 alignment data were also used to demarcate the partitions as used in this study. Partitions 210 corresponding to the exons of HspB1, HspB5, and HspB8 were separated according to the ENSEMBL 211 web site ${ }^{11}$.

\section{Determination of the evolutionary pressure on full-length sequences and sequence partitions}

213 In addition to the evaluation of selection at the level of individual codon sites, selection at the level of

214 the coding regions of the sHSPs as a whole (full-length sequences) or at the level of the partitions

$13 \overline{11 \text { https://useast.ensembl.org/index.html?redirect }=\text { no }}$ 
215 (corresponding approximately to the NTR, CeR, aCD, CTE) was also evaluated. The goal of this

216 partition-level selection analysis was to determine the selective pressure on defined regions relative to

217 the other regions of that same sHSP. Additionally, the collected partition-level data permit the

218 comparison of homologous partitions between the various sHSPs. Also, partitions corresponding to the

219 exon structure of these sHSPs were compared to provide insight into the evolutionary history of the

220 structure of these genes. Aggregate estimates of the omega values $(\bar{\omega})$ were calculated using the

221 standard MG94xREV codon substitution model implemented in the FitMG94 algorithm ${ }^{12}$ (Kosakovsky

222 Pond et al. 2010) and represent either the full-length sequence-wide inference or the partition-wide

223 inference of the alignment-wide omega $(\omega)$ values (point estimates) with associated confidence

224 intervals, an upper and lower bound. This approach provides a quantitative measure of the selective

225 pressures historically applied to each partitioned region on average. The calculated alignment-

226 wide aggregate $\bar{\omega}$-values are listed in Table S4 with upper and lower bounds, and are marked by

227 indices corresponding either to the full-length sequences or to the various partitions, e.g. $\bar{\omega}_{\mathrm{FL}}, \bar{\omega}_{\mathrm{NTD}}$,

228 etc. To test for significantly different aggregate $\bar{\omega}$ estimates, we performed a one degree of freedom

229 likelihood ratio test where the null hypothesis enforced the same aggregate value on both genes or

230 partitions, and the alternative hypothesis estimated them separately (FitMG94-Compare). Statistical

231 relationships with significantly different full-length or partitioned sequences, as identified by this

232 analysis, are indicated in the Hasse diagrams in Figs. 2 through 4 by arrows, pointing towards the

233 sequences with the greater numeric aggregate $\bar{\omega}$ values, i.e., with less stringent purifying selection.

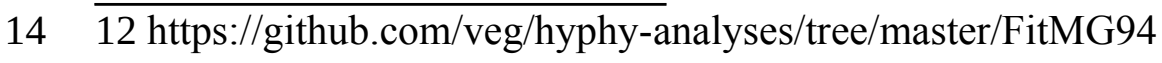




\section{Results}

\section{Selective pressure profiles along the human sHSP sequences}

236 The $\omega$-values (dN/dS point estimates) for each codon of the Gnathostomata HspB1, HspB3, HspB5, 237 and HspB8 alignments, as returned by the FEL model (Table S3), were plotted along the sequences of

238 the human sHSPs, omitting all codons which have no homologous sites in the human sequences (Fig.

239 1). Overall, these results reveal that most of the codons were exposed to varying degrees of purifying selection over their evolutionary history (no columns, black columns). A minor fraction of codons in

241 each sHSP do not reveal a detectable signal for purifying selective pressure, tentatively suggesting that

242 they have evolved neutrally (gray columns). Obviously, there are differences between these sHSPs in 243 terms of (1) the fraction of these neutrally evolving codons, (2) their distribution along the sHSPs 244 sequences, and (3) the strength of the detected selective pressures (see below). In HspB1 the codons 245 with neutral signature $(8 / 205 ; 3.9 \%)$ are distributed relatively evenly along the sequence with just one 246 cluster of two codons (T202, A203) found in the distal CTE (Fig. 1a). In HspB3 the fraction of 247 neutrally evolving codons is highest among the studied sHSPs $(28 / 150 ; 18.7 \%)$ with most of these 248 codons clustered in the CeR (Fig. 1b). Interestingly, HspB3 is the only studied sHSP with codons that 249 were historically exposed to a detectable positive diversifying selection (T48, V147), and not by chance 250 these codons are found in regions with less stringent conservation or even neutral evolution: in the CeR 251 and CTE. While in HspB5 the codons with neutral signature $(11 / 175 ; 6.3 \%)$ are relatively evenly 252 distributed over the entire sequence (Fig. 1c), in HspB8 these codons $(9 / 196 ; 4.6 \%)$ are clustered, to a 253 certain extent, in the CeR and CTE (Fig. 1d). 
HspB8, respectively) and in Table S4, in order to separate these sequence segments. These partitions correspond approximately to the domains and regions in the sHSPs as identified earlier by sequence alignments: NTR, $\alpha C D$, CeR and CTE (cf. Fig. S1). Such differentiation of the $\omega$-profile into regions with lower and higher $\omega$-values is most pronounced in HspB3 (Fig. 1b) and HspB8 (Fig. 1d) and to a

262 lesser extend in HspB1 (Fig. 1a). In HspB5 it is least pronounced (Fig. 1c) thus complicating the 263 allocation of the partitions. In regions with unclear differentiation (i.e., low vs. high $\omega$-values), 264 information from the sequence alignment (Fontaine et al. 2003; cf. Fig. S1) was also used for the delimitation of the partitions. The partitions as defined in Fig. 1 and Table S4 were used to quantitatively determine the selective pressures (measured as aggregate $\bar{\omega}$ ) to which the respective regions of these sHSPs were historically exposed to (see below).

\section{Differences in the selective pressures between the sHSPs (full-length sequences)}

Next, we attempted to quantitatively determine differences in the selective pressures acting over the evolutionary history of the Gnathostomata sHSPs, considering the full-length sequences. Based on the codon-specific $\omega$-values (dN/dS point estimates) as shown in Table S3, aggregate $\bar{\omega}$-values for each full-length sHSP ( $\bar{\omega}_{\mathrm{FL}}$; cf. Table S4) were inferred using the FitMG94 algorithm (Fig. 2a). Clearly, the obtained aggregate $\bar{\omega}_{\mathrm{FL}}$-values between these sHSPs differ: HspB8 and HspB3 were historically exposed to the most stringent (lowest $\bar{\omega}_{\mathrm{FL}}$ ) and most relaxed (highest $\bar{\omega}_{\mathrm{FL}}$ ) purifying selection, respectively, with $\mathrm{HspB} 1$ and $\mathrm{HspB} 5$ being placed in-between. Further statistical testing using the aggregate $\bar{\omega}_{\mathrm{FL}}$-values: (1) HspB3 evolved under a less stringent purifying selection compared to the other studied sHSPs; and (2) HspB1 evolved under a less stringent level of purifying selection than 
pressures between some of the sHSPs, as demonstrated here, may be related to the frequency by which

these sHSPs harbor disease-associated mutations (cf. Discussion).

\section{4}

\section{Differences in the selective pressures between the regions within the sHSPs}

285 In the next step we sought to verify that different parts of the four studied sHSP sequences were indeed counting the exon-specific partitions (see below).

Further testing using the FitMG94-Compare algorithm reveals the high statistical confidence for 


\section{Differences in the selective pressures between the homologous regions of the four studied sHSPs}

306 We used the collected aggregate $\bar{\omega}$-values (Table S4) also for the comparison among the homologous 307 regions, as defined in Fig. 1 and Fig. S1, of the four studied sHSPs (Fig. 3a). Applying the FitMG94-

308 Compare algorithm provided the Hasse diagrams shown in Fig. 3c, resulting in these relationships:

309 The NTR, CeR, and aCD of HspB3 exhibit the least degrees of purifying selection when compared to

310 the homologous partitions of the other sHSPs, while the CTE of HspB3 exhibits a lesser degree of

311 purifying selection when compared to the homologous partitions of HspB1 and HspB5, but not of

312 HspB8. The $\alpha \mathrm{CD}$ of HspB8 was exposed to a stronger purifying selection than the aCDs of all other

313 sHSPs. Quite differently, the CTE of HspB8 was exposed to a lesser degree of purifying selection than

314 the CTE of HspB1. None of the partitions exhibits a significant difference between HspB1 and HspB5,

315 a finding that is consistent with the fact that we could not detect a significant difference between the

316 full-length sequences of both proteins (cf. Fig. 2b). Similarly, most of the partitions (NTR, CeR, CTE)

317 do not exhibit a difference between HspB5 and HspB8, except the aCD which clearly exhibits a

318 difference. This difference, however, does not have a sufficient weight to cause a difference at the level

319 of the full-length sequences (cf. Fig. 2b). In summary, several significant differences in the degrees of

320 purifying selection between the homologous domains and regions of the studied sHSPs were detected.

\section{Differences in the selective pressures between the exons}

$322 \mathrm{HspB} 1, \mathrm{HspB} 5$ and HspB8 are encoded by genes consisting of three exons, whereas HspB3 is encoded by a one-exon gene (cf. Fig. 1). In HspB1, HspB5 and HspB8, exon 2 encodes the core part of the aCD, the most conserved region in these sHSPs (Fontaine et al. 2003; Franck et al. 2004). Applying the FitMG94 algorithm, we used the codon-specific $\omega$-values (dN/dS point estimates; Table S3) and inferred the aggregate $\bar{\omega}$-values for each exon-specific partition. As expected, plotting the exon- 
329 HspB5 exhibiting the least pronounced effect (Fig. 4a; cf. Table S4). When the FitMG94-Compare

330 algorithm was applied, this finding was confirmed with a high statistical confidence (Fig. 4b). This

331 analysis reveals also that exons 1 of $\mathrm{HspB} 1$ and HspB8 were under a more stringent purifying selection

332 than exons 3 of the same sHSPs. In HspB5, no such difference between exons 1 and 3 can be

333 demonstrated. When the homologous exons among the three sHSPs were compared, the FitMG94-

334 Compare algorithm did not detect such differences which would hold out against this level of statistical

335 confidence, with one exception: Exon 2 of HspB5 was historically exposed to a less stringent selective

336 pressure than exons 2 of $\mathrm{HspB} 1$ and $\mathrm{HspB} 8$ (Fig. 4c). This relation is noteworthy and may have

337 consequences for the frequency by which exons 2 in these sHSPs harbor disease-associated mutations

338 (cf. Discussion). In summary, the various exons of HspB1, HspB5, and HspB8 were historically

339 exposed to differential degrees of purifying selection. This relationship is most pronounced when

340 exons 2 are compared with exons 1 and 3 within the same sHSP on the one hand and among one

341 another on the other hand.

\section{Selective pressure on the missense mutation sites}

343 Based on the used set of Gnathostomata orthologs of HspB1, HspB3, HspB5 and HspB8, purifying

344 selection was detected at all mutation sites harboring missense mutations (cf. Fig. 1, Table S3; with the

345 relevant data summarized in Fig. 5). For most of these mutation sites, evidence for highly stringent

346 purifying selection was found with $\mathrm{dN} / \mathrm{dS}$ point estimates $(\omega$-values) of zero or near zero $(\omega<0.05)$.

347 The range of the $\omega$-values for the remaining mutation sites is $<0.35$, indicating lesser stringent

348 purifying selection. The four known mutation sites that harbored missense mutations which are

349 associated with recessive disease phenotypes (G53, S86, L99 in HspB1; R56 in HspB5) all exhibit $\omega$ -

350 values $<0.05$, indicating highly stringent purifying selection.

351 Interestingly, there is a disparate distribution of the known disease-associated missense

352 mutations among the exons in the various sHSPs: Exons 2 in HspB1 and HspB8 harbored a significant 
353 fraction of the known mutations in the respective sHSPs, whereas exon 2 in HspB5 harbored only a

354 minor faction (just one mutation), among all mutations known to date (Fig. 5). The reason for this

355 disparate distribution is not known, although it may be related to the relatively relaxed purifying 356 selection seen in exon 2 of HspB5 (cf. Fig. 4; see Discussion).

\section{Discussion}

358 In this study, we explore the evolutionary history of four of the ten human sHSPs: HspB1, HspB3,

359 HspB5, and HspB8. These sHSPs are known disease factors as mutant alleles have been found

360 associated with various forms of neuropathies, myopathies, and with cataracts in the lens of the eye.

361 We found that the Gnathostomata sHSPs have been generally under strong purifying selection that is 362 typical of highly conserved and functionally constrained genes. Interestingly, we observed varying 363 degrees of purifying selection across the four sHSPs (highest: HspB8; lowest: HspB3; HspB1 and 364 HspB5 being placed in between) (cf. Fig. 2). When the human sequences were partitioned into defined 365 segments with relatively higher and lower selective pressures (corresponding approximately to the 366 domains and regions of NTR, CeR, aCD, and CTE), our results demonstrate the highest degree of 367 purifying selection in the aCD and less stringent purifying selection in the flanking regions, with the 368 CeR and the CTE exhibiting the least degree of purifying selection. This finding is consistent with 369 earlier comparative studies based on sequence alignments (Fontaine et al. 2003; Franck et al. 2004;

370 Kriehuber et al. 2010). One finding of our study is that the obtained profiles of the strength of the 371 selective pressures are characteristic attributes for the studied sHSPs.

373 clear positive, diversifying selection (cf. Fig. 1b). The reason for this observation is not known. 
HspB5 was subject to the least stringent purifying selection (cf. Fig. 4). Interestingly, the regions of stringent purifying selection do not necessarily end at the borders of exon 2 and can extend into the nearby regions of exons 1 and 3. This is best seen with HspB8 (cf. Fig. 1d). stringent purifying selection (cf. Fig. 5). Two further observations are noteworthy: (1) All mutation sites in the aCD of HspB3 and HspB8, and three out of four mutations sites in the aCD of HspB5, exhibit only synonymous variation $(\omega=0)$, as opposed to most mutation sites in the aCD of HspB1 that exhibit both, synonymous and non-synonymous variations; and (2) mutation sites positioned in the CTEs of HspB1 and HspB5 exhibit, by trend, weaker purifying selective pressure than mutation sites in the other partitions.

Our results provide a partial explanation for some of the unusual features of the sHSPs, including the dimorphic pattern of their evolution, the observed differences in the mutation rates between the sHSPs in humans, and the prevalence of genetically dominant disease phenotypes resulting from gain-of-function mutations. These features are discussed in detail below:

(1) The dimorphic pattern of sHSP evolution. As determined earlier by phylogenetic analysis

391 (Kriehuber et al. 2010), the evolutionary history of the sHSPs with the dimorphic pattern exhibits a 392 striking feature: While the isolated central part of these proteins, the aCD, evolved along the species 393 tree across all domains of life from the beginning, the flanking regions (NTR + CeR, CTE) have trees 394 different from the aCD being consistent with extensive remodeling and complex evolutionary dynamics independently of the aCD. The same analysis revealed also that all Metazoa (including by gene duplication mostly after the separation of the different Bilateria taxa during the Cambrian species explosion. Interestingly, certain characteristic N-terminal sequences found otherwise scattered

399 all over the aCD-based phylogenetic tree and which served to establish the evolutionary dimorphic 
401 this context, our collected data provide an argument for the extension of this dimorphic evolutionary

402 pattern also to the branch of the Gnathostomata sHSPs. Differences in the selective pressures

403 (measured as aggregate $\bar{\omega}$-values) between the aCD with its most stringent purifying selection on the

404 one hand and the flanking regions (NTR + CeR, CTE) on the other hand with their more relaxed 405 purifying selection imply that this dimorphic evolutionary pattern continued also in the taxon 406 Gnathostomata (cf. Fig. 3b).

A closer inspection reveals also certain differences in the selective pressures between the different partitions of the flanking regions in all four studied sHSPs. For example, the purifying selective pressures in the NTR was historically stronger than in the CeR and CTE of all four studied sHSPs (cf. Fig. 3b). These differential selective pressures likely played a role in shaping the vertebrate sHSPs, even if the specifics are not understood at this time. The importance of these flanking regions for both the structure and function of the mammalian sHSPs has been demonstrated (Selig et al. 2020). in the references therein, sHSPs harbor disease-associated mutations with different incidences. This is especially evident if $\mathrm{HspB} 1, \mathrm{HspB} 3$, and $\mathrm{HspB} 8$ are compared, all of them causing, when mutated, a similar spectrum of peripheral motor neuropathies. HspB1 harbored by far most of the mutations as 417 compared to HspB3 and HspB8: In a systematic study of a cohort of 510 unrelated index patients with 418 peripheral neuropathies, 32 patients were found to have mutations in sHSPs $(32 / 510 ; 6.3 \%)$ (Echaniz419 Laguna et al. 2017). Among them, 28 patients carried mutations in HspB1, four patients in HspB8, and none in HspB3. Thus, in the patient population of this study, HspB1 is approximately seven times 421 more frequently affected than HspB8, while HspB3 apparently plays only a marginal role, if any. In another study of 163 patients belonging to 108 families with distal hereditary motor neuropathies, a 423 genetic diagnosis was achieved in $37 / 108$ families $(34.2 \%)$ or in $78 / 163$ of all patients (47.8\%) 424 (Frasquet et al. 2021). Among them, the most frequent cause of disease was mutations in HspB1, 425 besides mutations in several other genes. In that study, no mutations were found in HspB8 or HspB3. 
426 Similarly, in other systematic studies with smaller patient cohorts, a number of mutations in HspB1

427 were found, but none in the other sHSPs (Capponi et al. 2011; Luigetti et al. 2016).

This disparate incidence may be directly related to the different degrees of purifying selection

429 these sHSPs have been historically subjected to (measured as aggregate $\bar{\omega}$-values for the full-length

430 sequences $\left.\left(\bar{\omega}_{\mathrm{FL}}\right)\right)$. HspB3 and HspB8 were exposed to the most relaxed and most stringent purifying

431 selection, respectively, with HspB1 being placed in-between (cf. Table S4, Fig 2). Because of the

432 historically most stringent purifying selection, it is plausible that mutations in HspB8 would lead more

433 frequently to impaired fitness, thus eliminating the disease alleles over time from the human

434 population. Conversely, mutations in HspB3 may have fewer consequences for the fitness, thus

435 allowing the accumulation of mutated alleles in the population. If this were the case, there should exist

436 a relatively large number of $\mathrm{HspB} 3$ allelic variants in the healthy human population. HspB1,

437 positioned in-between, exhibits a certain degree of 'plasticity', allowing it to harbor mutations at an

438 increased rate compared to HspB8, although this comes with a cost for the health. However, since a

439 significant proportion of mutant HspB1-associated disorders have a late onset in life (references in

440 Table S1), these mutations are likely to come with relatively little fitness costs in the human population

441 in evolutionary terms. In this scenario, most mutant HspB1 alleles should be under weak purifying

442 selection in the human population, if any, thus permitting their accumulation.

444 with its mutations are quite different. However, in terms of both the estimated overall selective 445 pressure for full-length HspB5 (cf. Fig 2) and the number of the harbored missense mutations (cf. Fig.

446 5), HspB5 may share some similarity with HspB1. Yet, another feature is noteworthy: Exon 2 of

447 HspB5 harbored only a minor fraction (just one) of the known mutations, quite different from the exons

4482 of HspB1 and HspB8 that harbored a major fraction of the known mutations (cf. Fig. 5). This may be

449 related to the fact that exon 2 of $\mathrm{HspB} 5$ is distinguished by the relatively relaxed purifying selection it

450 was historically subjected to, compared to HspB1 and HspB8 (cf. Fig. 4a, c). Following the above 
451 logic, exon 2 of HspB5 may have a relatively high tendency to accumulate a number of allelic variants

452 in the healthy human population without major fitness costs in evolutionary terms.

(3) The prevailing of genetically dominant disease phenotypes resulting from gain-of-function

454 mutations. The fact that most missense mutations in sHSPs are associated with dominant (or semi-

455 dominant) disease phenotypes is contrary to the expectation, as mutagenesis studies in many organisms

456 revealed that $\sim 90 \%$ of the wild-type alleles are dominant over the mutant alleles, with dominant mutant

457 phenotypes being the exception in the wider picture (Wilkie 1994). Thus, in addition to the peculiar

458 dimorphic evolutionary history, sHSPs are also distinguished by their atypical genotype-phenotype

459 relation.

460 Heritable diseases in general are believed to persist in the human population because of a

461 balance between mutations, genetic drift, and natural selection, the latter eventually eliminating the

462 mutation from the population by purifying selection (Blekhman et al. 2008). For alleles causing highly

463 penetrant Mendelian disorders, purifying selection can be quite strong, unless the disease outbreak has

464 a late onset as is the case with most mutant sHSP-associated disorders (see references in Table S1).

465 Thus, in the human population the majority of the mutant sHSP alleles can be expected to be under

466 reduced purifying selection, as the late onset is unlikely to impose major fitness costs in evolutionary

467 terms. This is, however, not in conflict with the fact that all sHSP mutation sites themselves were

468 exposed to a stringent purifying selection, together with the entire genes, during the Gnathostomata

469 evolution, as we show here. Indeed, it was observed that Mendelian disease genes in general are under

470 a more widespread and stronger purifying selection if the disease-causing alleles are dominant, as

471 compared both to recessive and more complex disease genes (Blekhman et al. 2008). While this interrelation is highly interesting, the mechanisms behind it still await their elucidation. 
purifying selection. These facts and observations offer an explanation for both the high proportion of

477 dominant mutations among neuromuscular disease-associated sHSP mutations, and also for the 478 relatively high proportion of sHSP mutations (6.3\% and 10.4\%; Echaniz-Laguna et al. 2017; Frasquet 479 et al. 2021; respectively) among all mutations in patients with inherited peripheral neuropathies.

Historically the phenomenon of genetic dominance has been fiercely debated (summarized in

Benndorf et al. 2014). One aspect is that the existence of multiple paralogous genes, such as the 10

sHSP genes in humans, seems to be related to their receptivity to harboring dominant-negative

mutations. The evolutionary retention of such paralogs in polyploid species, or species with polyploid

ancestry that includes Homo sapiens, was explained by gene dosage effects and dominant-negative effects involving supramolecular structures (Comai 2005; Veitia 2010; Veitia and Birchler 2010). Such

oligomeric complexes are indeed a hallmark of all studied sHSPs (Mymrikov et al. 2011; Boelens

\section{7} 491 Arguments come from HspB1-, HspB5-, and HspB8-knock-out rodents that do not reproduce overt 492 disease phenotypes as are seen for the dominant missense mutations (Brady et al. 2001; Huang et al.

Mutations associated with dominant phenotypes fall into two categories: loss-of-function type (i.e., haploinsufficiency) and gain-of-function type (Wilkie 1994), although mixed types might also occur. For the dominant sHSP mutations, a loss-of-function situation can be tentatively dismissed. 2007; Bouhy et al. 2018), despite the fact that some impaired cell biological pathways, e.g. the autophagosome formation, could be identified (Haidar et al. 2019). Additional biochemical evidence supports this notion: Mutations in sHSPs do not necessarily result in the loss of key functions such as chaperoning and anti-apoptotic activities (Krishnan et al. 2008; Almeida-Souza et al. 2010), even if some modification, e.g. of the chaperoning specificity, may occur (Weeks et al. 2018). These findings tentatively suggest that loss-of-function mechanisms may not be the primary mechanism responsible for the manifestation of the disorders. 
501 mechanisms that seem to accommodate most situations (Wilkie 1994): (1) 'classic' dominant negative

502 effects (e.g. through protein-protein interactions); (2) formation of toxic gene products (e.g. of

503 amyloids); (3) impact on cytoskeletal systems resulting in altered cell architecture; and (4) increased

504 enzymatic activities. Existing experimental, genetic and clinical evidence suggests that most if not all

505 studied dominant disease-associated sHSP mutant alleles fit this pattern. Table S5 summarizes some of

506 the molecular and cellular consequences of the expression of dominant mutant sHSP alleles, all

507 supporting gain-of-function mechanisms. They result in abnormal protein-protein interactions and in

508 abnormal quaternary structures (implying dominant negative effects, summarized e.g. for a number of

509 HspB1 mutants by Muranova et al. (2020)), in the deposition of protein aggregates (implying the

510 formation of highly toxic amyloid precursors and altered cell architecture), and in increased

511 downstream enzymatic activities. This situation should have direct consequences for any future

512 therapeutic interventions which should aim to reduce these toxic effects of the mutated proteins, e.g. by

513 reducing the expression of the mutant proteins, by 'detoxification' of amyloid precursors of the

514 aggregates, or by inhibition of the abnormally increased downstream enzymatic activities. The latter

515 point was experimentally addressed by d'Ydewalle et al. (2011) who found that expression of mutant

516 HspB1 decreased the abundance of acetylated a-tubulin in mice that was paralleled by axonal transport

517 deficits. A pharmacological inhibition of the histone deacetylase 6 restored the a-tubulin acetylation

518 together with the axonal transport, and thus rescued the disease phenotype. Also, pharmacological

519 targeting sHSPs and their abnormal interactions with other proteins resulting from mutations is within

520 reach of future approaches (Boelens 2020).

521 Taken together, our data shed light onto some unusual features of the evolution of four of the

522 vertebrate sHSPs (HspB1, HspB3, HspB5, HspB8), and how their evolutionary history has shaped

523 these proteins. We provide evidence that the vertebrate sHSPs exhibit a dimorphic evolutionary

524 pattern, with different degrees of purifying selection acting historically upon the aCD on the one hand 
525 and on the flanking regions on the other hand. When compared to one another, the four studied sHSPs

526 have been exposed to different degrees of purifying selection. These features have implications for the

527 role of these sHSPs in human health and disease and may explain the different incidences by which the

528 various sHSPs harbored disease-associated mutations in the human population and also the unusual

529 genotype-phenotype relation in patients with neuromuscular diseases resulting from missense

530 mutations in these sHSPs. Experimental findings and theoretical considerations support the prevalence

531 of toxic gain-of-function mechanisms for the manifestation of the mutant sHSP-associated diseases in

532 most individuals. We propose that any future therapeutic approaches should directly tackle these toxic

533 properties of mutant sHSPs, or of toxic downstream consequences of their expression. Examples

534 include the interference in abnormal protein-protein interactions involving mutant sHSPs, the inhibition

535 of the formation of toxic amyloids, the protection of the cytoskeletal architecture, or the inhibition of

536 abnormally increased downstream enzymatic activities.

537 Our study has demonstrated how the analysis of the evolution of vertebrate genes, which cause

538 inheritable diseases if mutated, has the potency to lead to future medical strategies. In so doing, we

539 provide support for Theodosius G. Dobzhansky's famous realization that 'nothing in biology makes

540 sense except in the light of evolution' (Dobzhansky 1973). The extension of this paradigm to

541 inheritable human diseases may help to advance the emerging field of 'Evolutionary Medicine'. 


\section{Literature}

543 Adriaenssens E, Geuens T, Baets J, Echaniz-Laguna A, Timmerman V (2017) Novel insights in the disease biology of

544 mutant small heat shock proteins in neuromuscular diseases. Brain 140:2541-2549

545 Almeida-Souza L, Goethals S, Winter V De, Dierick I, Gallardo R, Durme J Van, Irobi J, Gettemans J, Rousseau F,

546 Schymkowitz J, Timmerman V, Janssens S (2010) Increased monomerization of mutant HSPB1 leads to protein

547 hyperactivity in Charcot-Marie-Tooth neuropathy. J Biol Chem 285:12778-12786

548 Arenas M (2015) Trends in substitution models of molecular evolution. Front Genet 6:319

549 Benndorf R, Martin JL, Kosakovsky Pond SL, Wertheim JO (2014) Neuropathy- and myopathy-associated mutations in

550 human small heat shock proteins: characteristics and evolutionary history of the mutation sites. Mutat Res Rev Mutat

$551 \quad$ Res $761: 15-30$

552 Blekhman R, Man O, Herrmann L, Boyko AR, Indap A, Kosiol C, Bustamante CD, Teshima KM, Przeworski M (2008)

553 Natural selection on genes that underlie human disease susceptibility. Curr Biol 18:883-889

554 Boelens WC (2020) Structural aspects of the human small heat shock proteins related to their functional activities. Cell

$555 \quad$ Stress Chaperones 25:581-591

556 Bouhy D, Juneja M, Katona I, Holmgren A, Asselbergh B, De Winter V, Hochepied T, Goossens S, Haigh JJ, Libert C,

557 Ceuterick-de Groote C, Irobi J, Weis J, Timmerman V (2018) A knock-in/knock-out mouse model of HSPB8-associated

558 distal hereditary motor neuropathy and myopathy reveals toxic gain-of-function of mutant HspB8. Acta Neuropathol

$559 \quad 135: 131-148$

560 Brady JP, Garland DL, Green DE, Tamm ER, Giblin FJ, Wawrousek EF (2001) AlphaB-crystallin in lens development and

561 muscle integrity: a gene knockout approach. Invest Ophthalmol Vis. Sci. 42:2924-2934

562 Capponi S, Geroldi A, Fossa P, Grandis M, Ciotti P, Gulli R, Schenone A, Mandich P, Bellone E (2011) HSPB1 and HSPB8

563 in inherited neuropathies: study of an Italian cohort of dHMN and CMT2 patients. J Peripher Nerv Syst 16:287-294

564 Carra S, Alberti S, Benesch JLP, Boelens W, Buchner J, Carver JA, Cecconi C, Ecroyd H, Gusev N, Hightower LE, Klevit

565 RE, Lee HO, Liberek K, Lockwood B, Poletti A, Timmerman V, Toth ME, Vierling E, Wu T, Tanguay RM (2019) Small

566 heat shock proteins: multifaceted proteins with important implications for life. Cell Stress Chaperones 24:295-308.

567 Chen J, Liu X, Xu Y, Fan D (2021) Rare variants of HSPB1 are probably associated with amyotrophic lateral sclerosis. Nan

$568 \quad$ Fang Yi Ke Da Xue Xue Bao 41:75-78

569 Comai L (2005) The advantages and disadvantages of being polyploid. Nat Rev Genet 6:836-846 
Datskevich PN, Nefedova VV, Sudnitsyna MV, Gusev NB (2012) Mutations of small heat shock proteins and human congenital diseases. Biochemistry (Mosc) 77:1500-1514

de Jong WW, Caspers GJ, Leunissen JA (1998) Genealogy of the alpha-crystallin-small heat-shock protein superfamily. Int J Biol Macromol 22:151-162

Dobzhansky TG (1973) Nothing in biology makes sense except in the light of evolution. Am Biol Teacher 35:125-129

d'Ydewalle C, Krishnan J, Chiheb DM, Van Damme P, Irobi J, Kozikowski AP, Vanden Berghe P, Timmerman V, Robberecht W, Van Den Bosch L (2011) HDAC6 inhibitors reverse axonal loss in a mouse model of mutant HSPB1induced Charcot-Marie-Tooth disease. Nat Med 17:968-974

Echaniz-Laguna A, Geuens T, Petiot P, Péréon Y, Adriaenssens E, Haidar M, Capponi S, Maisonobe T, Fournier E, Dubourg O, Degos B, Salachas F, Lenglet T, Eymard B, Delmont E, Pouget J, Juntas Morales R, Goizet C, Latour P, Timmerman V, Stojkovic T (2017) Axonal Neuropathies due to Mutations in Small Heat Shock Proteins: Clinical, Genetic, and Functional Insights into Novel Mutations. Hum Mutat 38:556-568

Fontaine JM, Rest JS, Welsh MJ, Benndorf R (2003) The sperm outer dense fiber protein is the 10th member of the superfamily of mammalian small stress proteins. Cell Stress Chaperones 8:62-69

Franck E, Madsen O, van Rheede T, Ricard G, Huynen MA, de Jong WW (2004) Evolutionary diversity of vertebrate small heat shock proteins. J Mol Evol 59:792-805

Frasquet M, Rojas-García R, Argente-Escrig H, Vázquez-Costa JF, Muelas N, Vílchez JJ, Sivera R, Millet E, Barreiro M, Díaz-Manera J, Turon-Sans J, Cortés-Vicente E, Querol L, Ramírez-Jiménez L, Martínez-Rubio D, SánchezMonteagudo A, Espinós C, Sevilla T, Lupo V (2021) Distal hereditary motor neuropathies: Mutation spectrum and genotype-phenotype correlation. Eur J Neurol. 28:1334-1343

Frost SD, Wrin T, Smith DM, Kosakovsky Pond SL, Liu Y, Paxinos E, Chappey C, Galovich J, Beauchaine J, Petropoulos CJ, Little SJ, Richman DD (2005) Neutralizing antibody responses drive the evolution of human immunodeficiency virus type 1 envelope during recent HIV infection. Proc Natl Acad Sci USA 102:18514-18519

Haidar M, Asselbergh B, Adriaenssens E, De Winter V, Timmermans JP, Auer-Grumbach M, Juneja M, Timmerman V (2019) Neuropathy-causing mutations in HSPB1 impair autophagy by disturbing the formation of SQSTM1/p62 bodies. Autophagy 15:1051-1068

Huang L, Min JN, Masters S, Mivechi NF, Moskophidis D (2007) Insights into function and regulation of small heat shock protein 25 (HSPB1) in a mouse model with targeted gene disruption. Genesis 45:487-501 
Kampinga HH, Hageman J, Vos MJ, Kubota H, Tanguay RM, Bruford EA, Cheetham ME, Chen B, Hightower LE (2009) Guidelines for the nomenclature of the human heat shock proteins. Cell Stress Chaperones 14:105-111

Kappé G, Boelens WC, de Jong WW (2010) Why proteins without an alpha-crystallin domain should not be included in the human small heat shock protein family HSPB. Cell Stress Chaperones 15:457-461

Kappé G, Franck E, Verschuure P, Boelens WC, Leunissen JA, de Jong WW (2003) The human genome encodes 10 alphacrystallin-related small heat shock proteins: HspB1-10. Cell Stress Chaperones 8:53-61

Katz M, Davis M, Garton FC, Henderson R, Bharti V, Wray N, McCombe P (2020). Mutations in heat shock protein beta-1 (HSPB1) are associated with a range of clinical phenotypes related to different patterns of motor neuron dysfunction: A case series. J Neurol Sci 413:116809

Kosakovsky Pond SL, Frost SD (2005) Not so different after all: a comparison of methods for detecting amino acid sites under selection. Mol Biol Evol 22:1208-1222

Kosakovsky Pond S, Delport W, Muse SV, Scheffler K (2010) Correcting the Bias of Empirical Frequency Parameter Estimators in Codon Models. PLoS One 5(7): e11230

Kriehuber T, Rattei T, Weinmaier T, Bepperling A, Haslbeck M, Buchner J (2010) Independent evolution of the core domain and its flanking sequences in small heat shock proteins. FASEB J 24:3633-3642

Krishnan J, D’Ydewalle C, Dierick I, lrobi J, van den Berghe P, Janssen P, Timmerman V, Robberecht W, van den Bosch L (2008) Pathogenic mechanisms of HSPB1 mutations associated with distal hereditary motor neuropathies. In: 38 th Annual Meeting of the Society for Neuroscience, November 15-19, Washington, DC

Kristensen DM, Wolf YI, Mushegian AR, Koonin EV (2011). Computational methods for Gene Orthology inference. Brief Bioinform 12:379-391

Kuraku S, Feiner N, Keeley SD, Hara Y (2016) Incorporating tree-thinking and evolutionary time scale into developmental biology. Dev Growth Differ 58:131-142

Luigetti M, Fabrizi GM, Bisogni G, Romano A, Taioli F, Ferrarini M, Bernardo D, Rossini PM, Sabatelli M (2016) CharcotMarie-Tooth type 2 and distal hereditary motor neuropathy: Clinical, neurophysiological and genetic findings from a single-centre experience. Clin Neurol Neurosurg 144:67-71

Muranova LK, Sudnitsyna MV, Strelkov SV, Gusev NB (2020) Mutations in HspB1 and hereditary neuropathies. Cell Stress Chaperones 25:655-665

Mymrikov EV, Seit-Nebi AS, Gusev NB (2011) Large potentials of small heat shock proteins. Physiol Rev 91:1123-1159

Pond SL, Frost SD, Muse SV (2005) HyPhy: hypothesis testing using phylogenies. Bioinformatics 21:676-679 
Pond SL, Frost SD (2005) Datamonkey: rapid detection of selective pressure on individual sites of codon alignments.

Price, M.N., Dehal, P.S., and Arkin, A.P. (2010) FastTree 2 - Approximately Maximum-Likelihood Trees for Large

631 Quintana-Murci L, Barreiro LB (2010) The role played by natural selection on Mendelian traits in humans. Ann NY Acad

\section{Sci 1214:1-17}

633 Selig EE, Zlatic CO, Cox D, Mok YF, Gooley PR, Ecroyd H, Griffin MDW (2020) N- and C-terminal regions of alphaB-

634 crystallin and Hsp27 mediate inhibition of amyloid nucleation, fibril binding, and fibril disaggregation. J Biol Chem

$635 \quad 295: 9838-9854$

636 Veitia RA (2010) A generalized model of gene dosage and dominant negative effects in macromolecular complexes. FASEB $637 \quad$ J 24:994-1002

638 Veitia RA, Birchler JA (2010) Dominance and gene dosage balance in health and disease: why levels matter! J Pathol. 220 : $639 \quad 174-185$

640 Vendredy L, Adriaenssens E, Timmerman V (2020) Small heat shock proteins in neurodegenerative diseases. Cell Stress

\section{Chaperones 25:679-699}

642 Wang K, Gawinowicz MA, Spector A (2000) The effect of stress on the pattern of phosphorylation of alphaA- and alphaB-

$643 \quad$ crystallin in the rat lens. Exp Eye Res. 71:385-393

644 Webster JM, Darling AL, Uversky VN, Blair LJ (2019) Small Heat Shock Proteins, big impact on protein aggregation in 645 neurodegenerative disease. Front Pharmacol 10:1047

646 Weeks SD, Muranova LK, Heirbaut M, Beelen S, Strelkov SV, Gusev NB (2018) Characterization of human small heat 647 shock protein HSPB1 alpha-crystallin domain localized mutants associated with hereditary motor neuron diseases. Sci $648 \quad \operatorname{Rep~8:688}$

649 Wilkie AO (1994) The molecular basis of genetic dominance. J Med Genet 31:89-98 


\section{Figure legends}

651 Fig. 1 Selective pressures along the sequences of human HspB1 (a), HspB3 (b), HspB5 (c) and

652 HspB8 (d) as returned by the FEL algorithm. The dN/dS point estimates were determined for each

653 codon of the aligned Gnathostomata sHSP orthologs, omitting all codons without homologous amino

654 acid residues in the human sequences. The plots show the principal $\omega$-values with the respective

655 confidence intervals (lower and upper bound; light gray error bars) along the primary sequences. No

656 columns or black columns: $\omega<1$, indicating that these positions were historically exposed to purifying

657 selection, using $\mathrm{p} \leq 0.1$ as statistical significance threshold; dark gray columns: $\omega \approx 1$ or near 1 , with $p$

$658>0.1$, indicating neutral evolution; blue columns: $\omega>1$, with $p<0.1$, indicating positive selection.

659 Positions with undefined $\omega$-values are indicated by columns of gray x. Amino acid residues affected

660 by disease-associated missense mutations are highlighted in color (red, green: dominant and recessive

661 disease phenotypes, respectively). Sequence segments (partitions) with relatively low, intermediate or

662 high $\omega$-values in average, as they can be discerned, are indicated and were used to delineate the 663 sequence partitions as used in this study (cf. Table S4). These partitions correspond approximately, 664 though not perfectly, to the NTR, CeR, aCD, and CTE. The exons are indicated where applicable.

665 Fig. 2 Selective pressures estimated for the full-length sHSP sequences. a, Plot of the dN/dS 666 estimates (aggregate $\bar{\omega}$-values with confidence intervals) of the full-length sHSPs, as returned by the 667 FitMG94 algorithm. The data were taken from Table S4. b, Hasse diagram, as inferred by the 668 FitMG94-Compare method, demonstrating the statistical relationship between the aggregate $\bar{\omega}$-values 669 of the four human sHSPs. The arrows point to the sHSPs with the higher $\bar{\omega}$-values, i.e., they were 670 historically exposed to a less stringent purifying selection. 
671 Fig. 3 Selective pressures estimated for the various sHSP domains and regions. a, Plot of the

$672 \mathrm{dN} / \mathrm{dS}$ estimates (aggregate $\bar{\omega}$-values with confidence intervals) of the region-specific partitions of the 673 four studied sHSPs, as returned by the FitMG94 algorithm. The data were taken from Table S4. For 674 comparison the aggregate $\bar{\omega}$-values of the full-length sequences are also included (gray columns). b, c, 675 Hasse diagrams, as determined by the FitMG94-Compare method, demonstrating the statistical 676 relationship between the aggregate $\bar{\omega}$-values of the regions within each sHSP sequence (b) or between 677 the homologous regions of the four sHSPs (c). The arrows are used as in Fig. 2.

678 Fig. 4 Selective pressures estimated for the exons of HspB1, HspB5, and HspB8. a, Plot of the $679 \mathrm{dN} / \mathrm{dS}$ estimates (aggregate $\bar{\omega}$-values with confidence intervals) of the various exon-specific partitions, 680 as returned by the FitMG94 algorithm. The data were taken from Table S4. For comparison, the 681 aggregate $\bar{\omega}$-values of the full-length sequences are also included (gray columns). b, c, Hasse 682 diagrams, as determined by the FitMG94-Compare method, demonstrating the statistical relationship 683 between the aggregate $\bar{\omega}$-values of the exons within each sHSP sequence (b) or between exons 2 of all 684 three sHSPs (c). The arrows are used as in Fig. 2.

685 Fig. 5 Summary of the selective pressures detected at the disease-associated missense mutation 686 sites of the four studied sHSPs. The dN/dS point estimates ( $\omega$-values with confidence intervals) for 687 each mutation site were taken from Table S3. The positions of the mutation sites within the various 688 sequence partitions (domains, regions, exons) of the sHSPs are indicated (cf. Table S1, Fig. 1). The 689 asterisks mark mutation sites whose mutations are associated with a recessive disease phenotype. All 690 other mutation sites are affected by mutations that are associated with a dominant disease phenotype, or 691 this association can be assumed. 


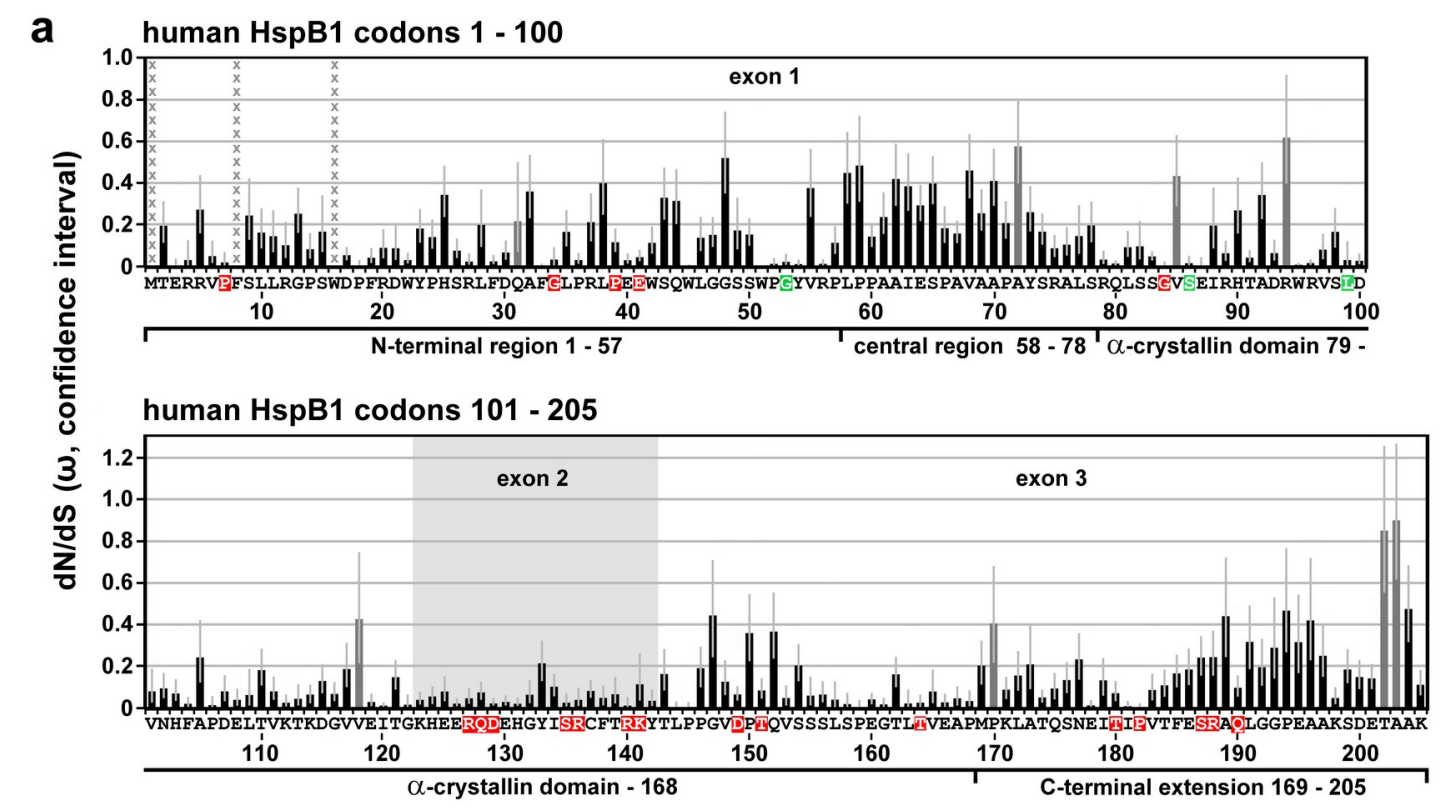

b human HspB3 codons 1 - 100

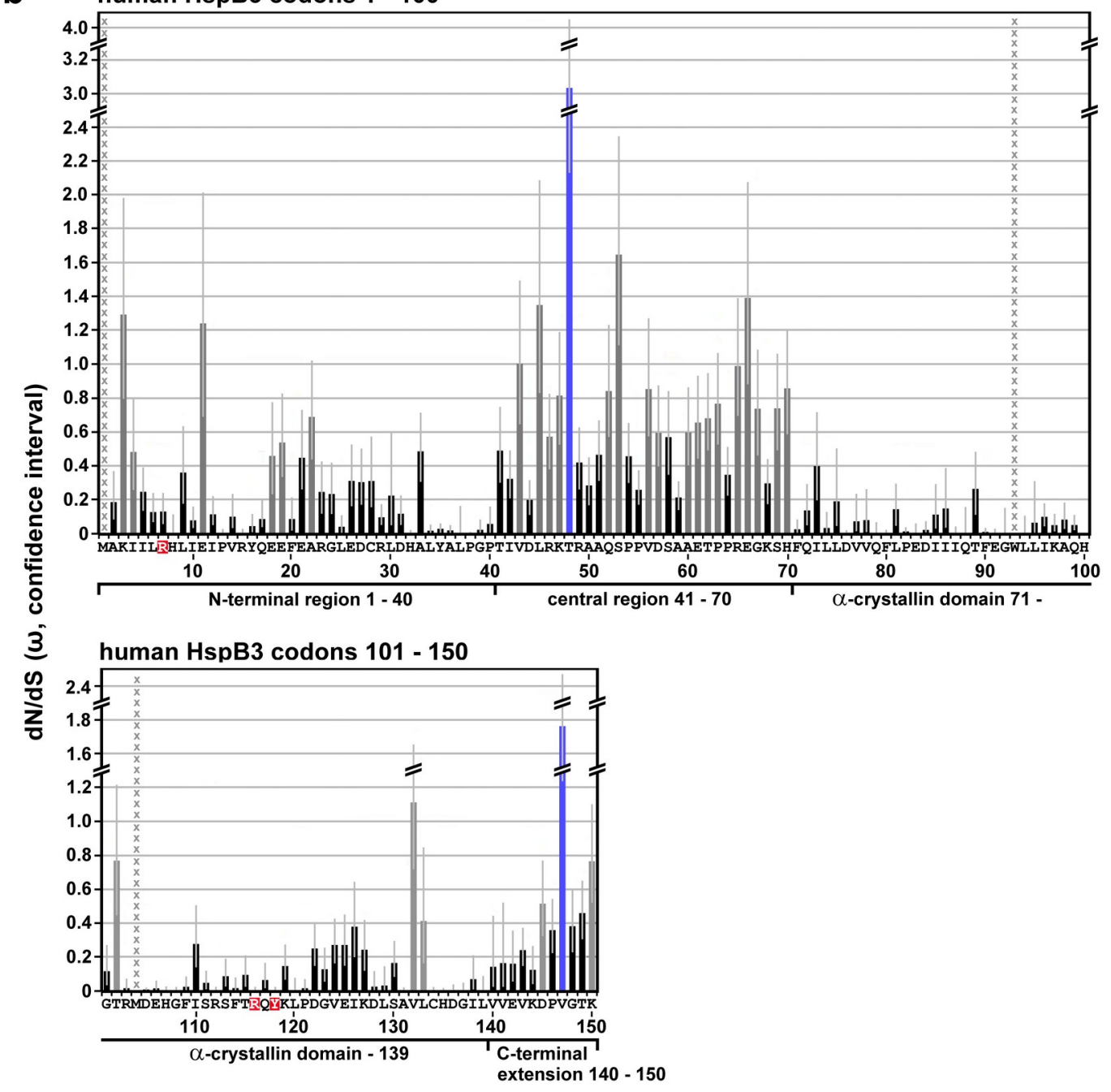

Figure 1 (part 1) 
C human HspB5 codons 1- 100

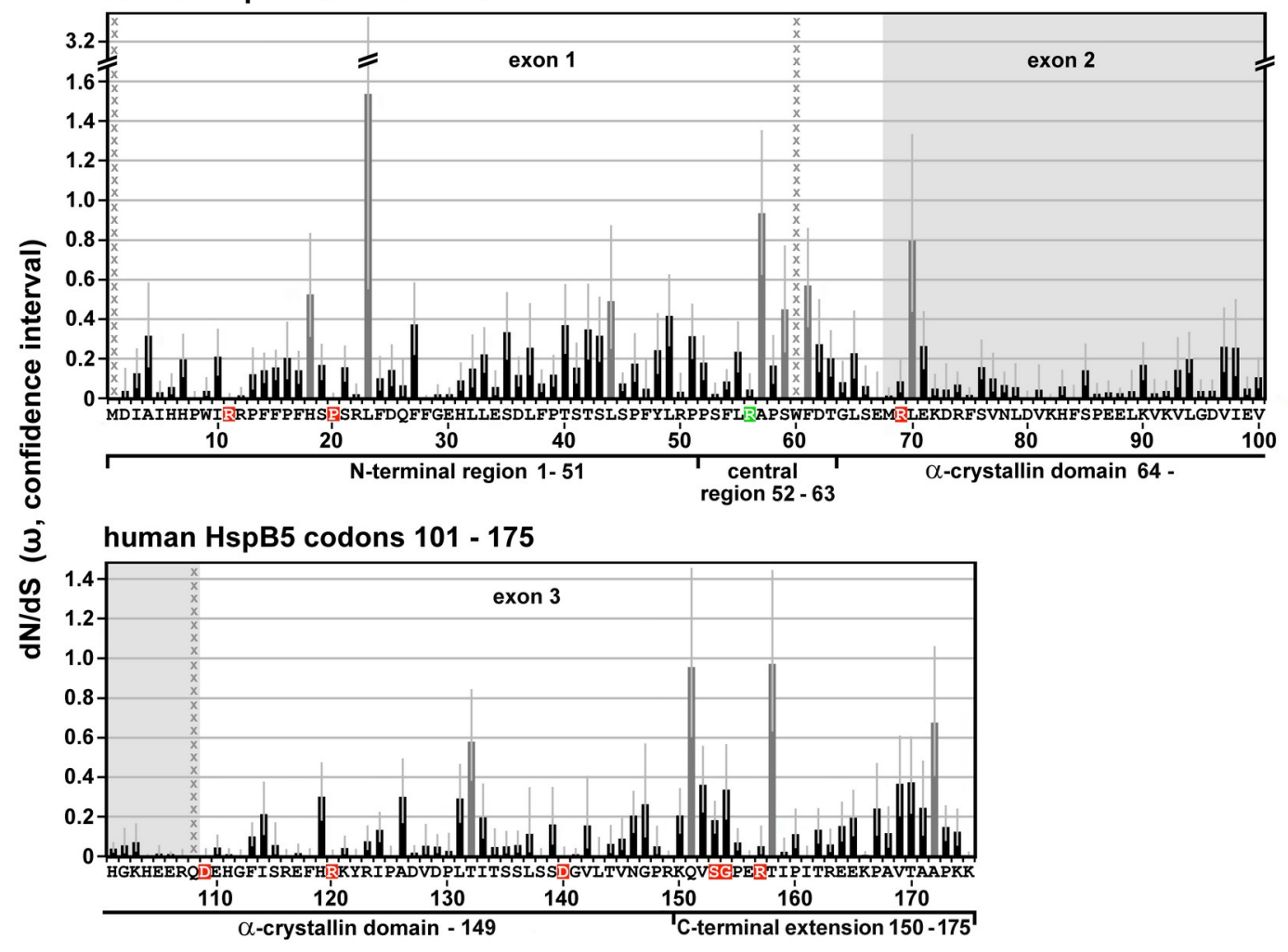

d human HspB8 codons $1-100$

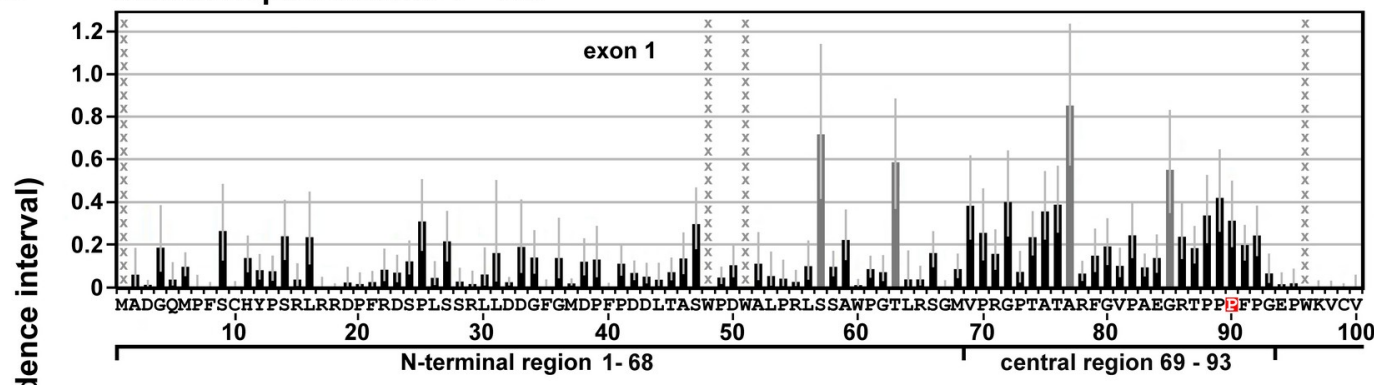

human HspB8 codons 101 - 196

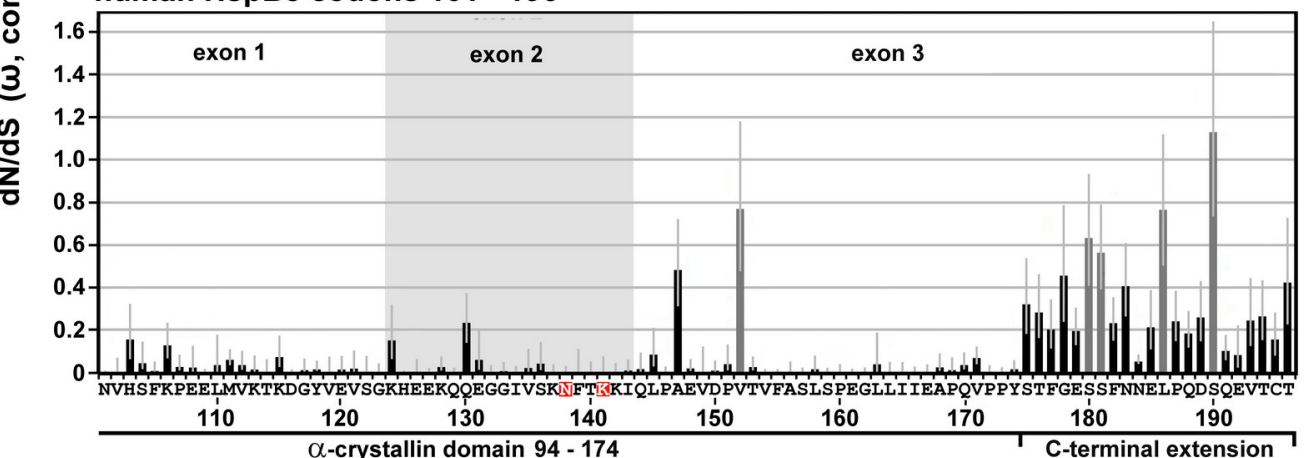

$175-196$

Figure 1 (part 2) 


\section{a selective pressures of} full-length sHSPs

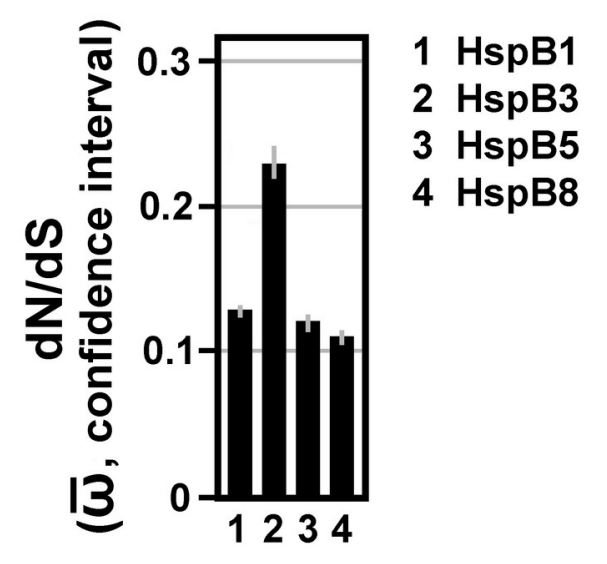

\section{b comparison of the sHSPs}

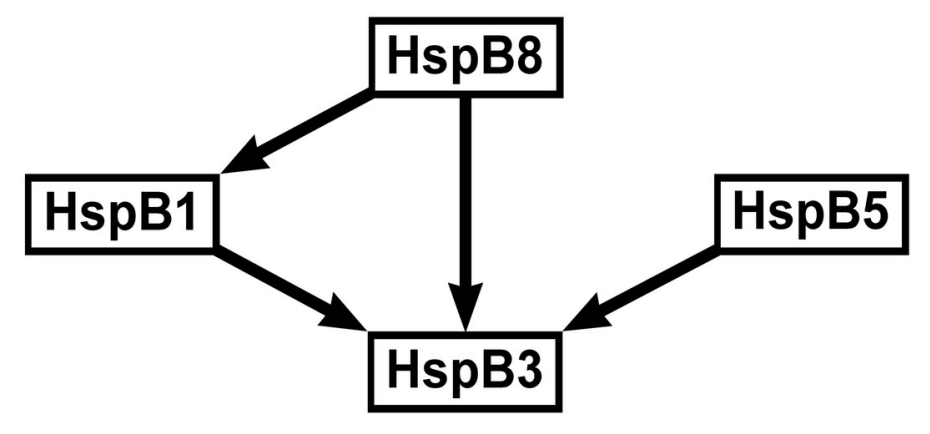

Figure 2 
a sHSP region-specific selective pressures

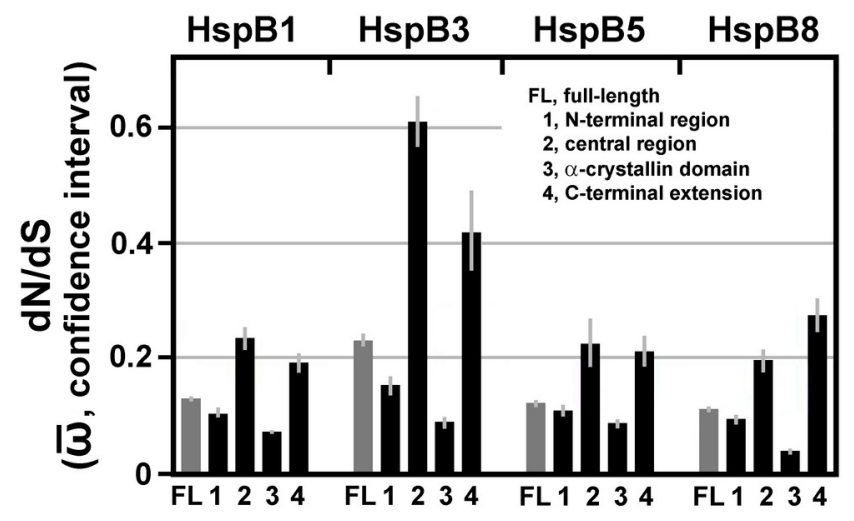

b comparison of the regions within each SHSP
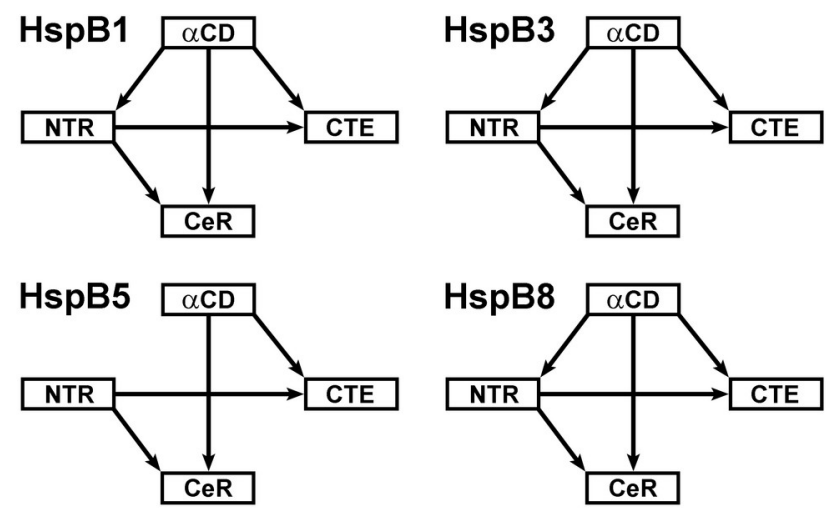

C comparison of the homologous regions

of HspB1, HspB3, HspB5 and HspB8
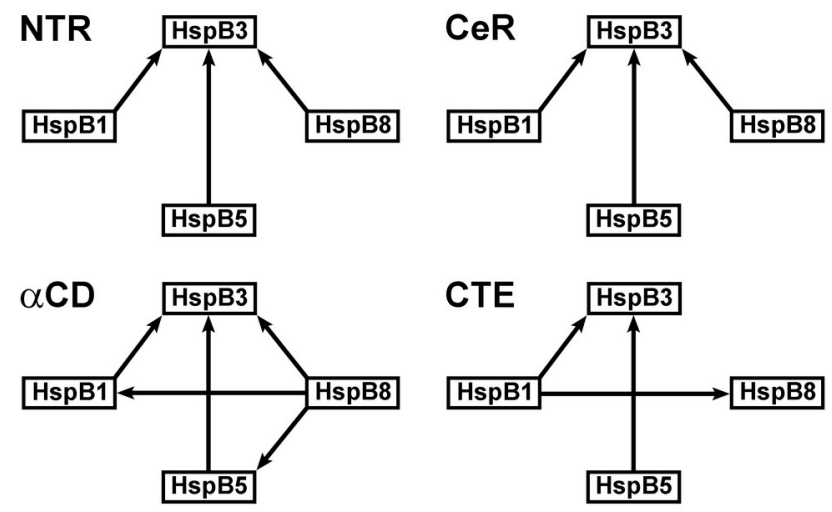

Figure 3 
a exon-specific selective pressures

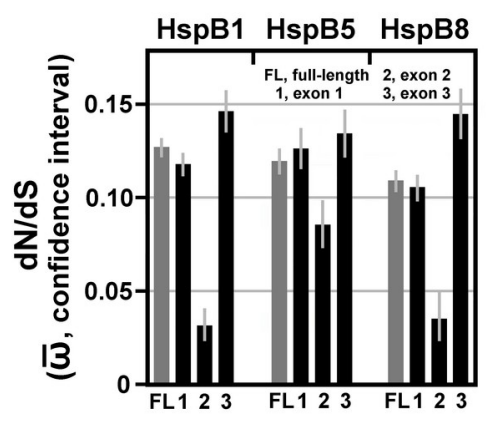

b comparison of the exons within each sHSP
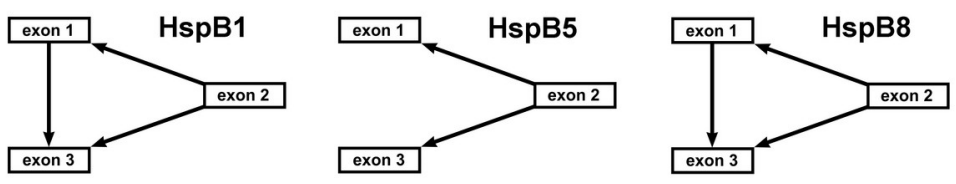

C comparison of exons 2 between HspB1, HspB5, and HspB8

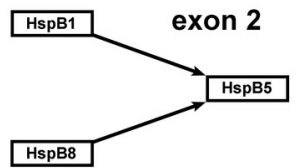

Figure 4
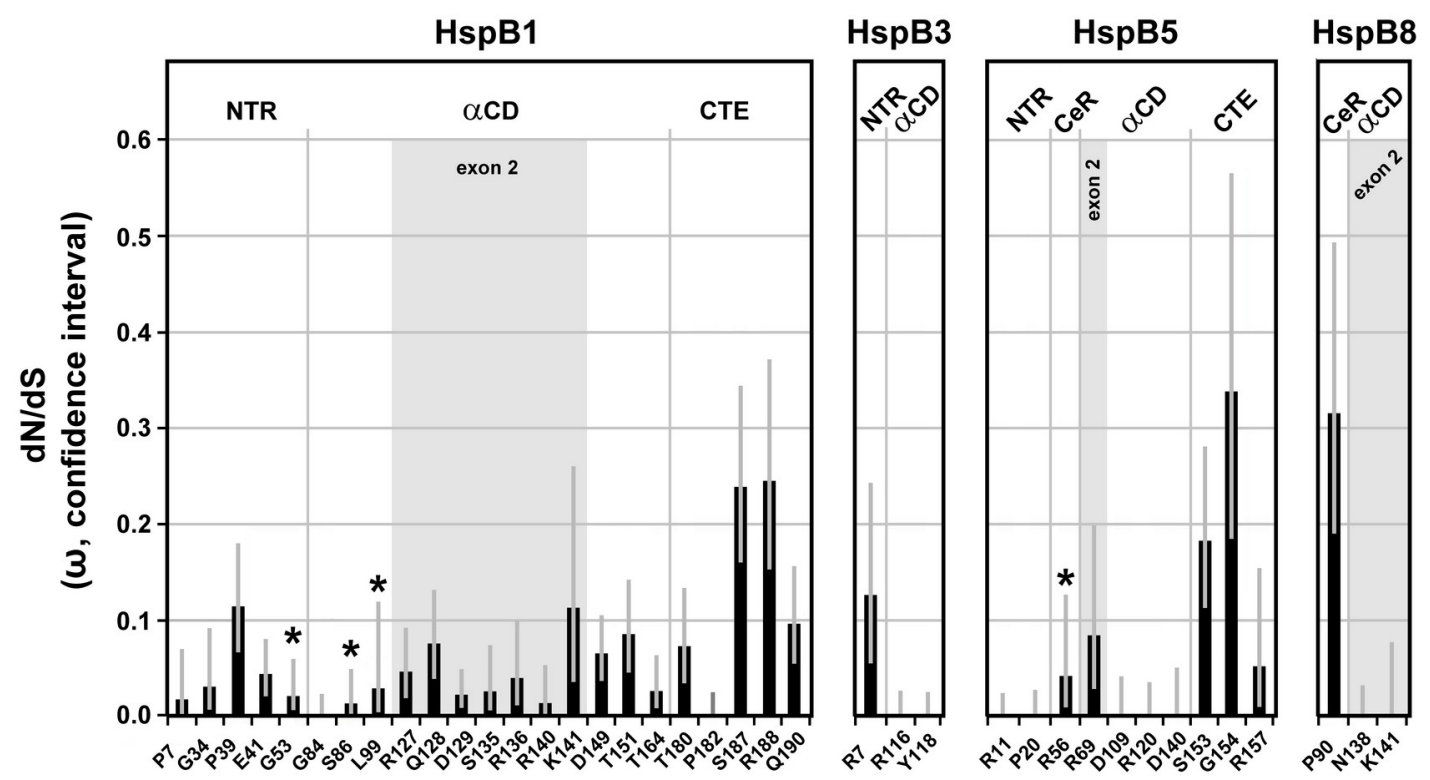

Figure 5 